\title{
Insufficient evidence for the effectiveness of ultrasonic instrumentation for root canal treatment
}

\author{
Is ultrasonic instrumentation more effective than hand instrumentation for \\ orthograde root canal treatment?
}

\author{
Sequeira P, Fedorowicz Z, Nasser M, Pedrazzi V. \\ Ultrasonic versus hand instrumentation for orthograde root canal \\ treatment of permanent teeth. Cochrane Database Syst Rev 2007; \\ issue 4
}

Data sources Relevant studies were sourced using the Cochrane Oral Health Group Trials Register, Cochrane Central Register of Controlled Trials (CENTRAL), Medline and Embase. The reference lists of relevant articles were searched and personal databases of trial reports used, in an attempt to locate additional published and unpublished trials. No language restriction was applied.

Study selection Randomised controlled trials (RCT) were selected that involved people of age $\geq 18$ years with single and multiple permanent teeth with a completely formed apex and no evidence of internal resorption, who required root canal treatment. Patients undertaking retreatment of a tooth were excluded.

Data extraction and synthesis Screening of eligible studies was conducted in duplicate and independently by two review authors. Results were to be expressed as fixed- or random-effects models using mean differences for continuous outcomes and risk ratios for dichotomous outcomes with $95 \%$ confidence intervals. Heterogeneity was to be investigated including both clinical and methodological factors.

Results No eligible RCT could be found.

Conclusions This review illustrates how there are no published or ongoing RCT relevant to this review question and that there is currently insufficient evidence regarding the effectiveness of ultrasonic instrumentation used alone for orthograde root canal treatment. Future RCT might focus more closely on evaluating the effectiveness of hand instrumentation compared with hand instrumentation and adjunctive ultrasonic instrumentation.

\section{Commentary}

Ultrasonic root canal instrumentation is regarded as an important adjunct to efforts to make endodontics predictable and efficient, at both surgical and nonsurgical levels. Currently, the practical importance of nonsurgical ultrasonic applications is in the management of complex problems in endodontics, including removal of broken instruments, chemical debridement or management of calcified canals. Surgically, ultrasonic retrotips procedures involving rootend preparation are now considered the gold standard for root-end surgical management.

The above review attempts to answer a valid question regarding the development of ultrasonic root canal files. In spite of the initial ex-vivo studies that showed promising results in this area, ${ }^{1}$ instrumentation by hand or rotary files is still considered to be the golden standard for root canal mechanical preparation. Visiting the relevant literature unearths considerable concerns regarding the current ultrasonic systems, including the temperature ${ }^{2}$ of the dentine and negotiating fine curvatures inside the root canals.

The clinical superiority of full orthograde ultrasonic instrumentation can be most effectively demonstrated by clinical studies. This report shows that there is not enough evidence to recommend using ultrasonics alone in the mechanical preparation of the root canal system. This does not, however, disqualify the significant applications of this technology in initial root canal instrumentation and retrograde preparation.

\section{Practice point}

At present, the evidence is not strong enough to recommend a move from manual and rotary files to ultrasonic systems.

\author{
Khaled Balto \\ King Abdulaziz University, Faculty of Dentistry, Jeddah, \\ Saudi Arabia \\ 1. Burleson A, Nusstein J, Reader A, Beck M. The in vivo evaluation of hand/rotary/ \\ ultrasound instrumentation in necrotic, human mandibular molars. J Endod 2007; \\ 33:782-787. \\ 2. Huttula AS, Tordik PA, Imamura G, Eichmiller FC, McClanahan SB. The effect \\ of ultrasonic post instrumentation on root surface temperature. J Endod 2006; \\ 32:1085-1087. \\ Evidence-Based Dentistry (2008) 9, 12. doi:10.1038/sj.ebd.6400563
}

